\title{
Volumetric Absorptive Microsampling as a Sampling Alternative in Clinical Trials and Therapeutic Drug Monitoring During the COVID-19 Pandemic: A Review
}

This article was published in the following Dove Press journal:

Drug Design, Development and Therapy

\author{
Yahdiana Harahap (D) \\ Rasmina Diptasaadya' \\ Denni Joko Purwanto (D) ${ }^{2}$ \\ 'Faculty of Pharmacy, Universitas \\ Indonesia, Depok, West Java 16424 , \\ Indonesia; ${ }^{2}$ Functional Medical Staff of \\ Surgical Oncology, Dharmais Cancer \\ Hospital, Jakarta II420, Indonesia
}

\begin{abstract}
An infectious disease, COVID-19, caused by a new type of coronavirus, has been discovered recently. This disease can cause respiratory distress, fever, and fatigue. It still has no drug and vaccine for treatment and prevention. Therefore, WHO recommends that people should stay at home to reduce disease transmission. Due to the quarantine, FDA stated that this could hamper drug development clinical trial protocols. Hence, an alternative sampling method that can be applied at home is needed. Currently, volumetric absorptive microsampling (VAMS) has become attention in its use in clinical and bioanalytical fields. This paper discusses the advantages and challenges that might be found in the use of VAMS as an alternative sampling tool in clinical trials and therapeutic drug monitoring (TDM) during the COVID-19 pandemic. VAMS allows easy sampling, can be done at home, storage and delivery at room temperature, and the volume taken is small and minimally invasive. VAMS is also able to absorb a fixed volume that can increase the accuracy and precision of analytical methods, and reduce the hematocrit effects (HCT). The use of VAMS is expected to be implemented immediately in clinical trials and TDM during this pandemic considering the benefits it has.
\end{abstract}

Keywords: clinical trials, COVID-19, drug development, therapeutic drug monitoring, TDM, volumetric absorptive microsampling, VAMS

\section{Introduction}

Recently, infectious disease has been found that is caused by a new type of coronavirus. This virus is called severe acute respiratory syndrome coronavirus 2 or SARS-CoV-2 and the disease it causes is called coronavirus disease 2019 or COVID-19. ${ }^{1}$ Most people infected with the SARS-CoV-2 virus will experience mild to moderate respiratory disease, fever, and fatigue. It can severely impact older people and people with congenital diseases. ${ }^{2}$ Experts believe that the primary disease transmission of this virus is from person to person. ${ }^{3}$ People can be infected if they make close contact (around 1-2 meters) with respiratory droplets of someone who has been infected. ${ }^{4}$ Based on the ongoing global transmission of COVID-19, WHO announced the disease as a pandemic on March 11, 2020, because there was no place that was completely safe from its effects. ${ }^{2}$ It was reported that this virus could stay alive for several hours in the air and several days on the surface of objects. ${ }^{5}$
Correspondence: Yahdiana Harahap Faculty of Pharmacy, Universitas Indonesia, Pondok Cina, Beji, Depok, West Java 16424, Indonesia

Phone/Fax +62 818222192/021

$78849001-003$

Email yahdiana03@yahoo.com 
According to the data collected by Johns Hopkins University in the United States, as of July 28, 2020, more than 16.489.485 total COVID-19 cases have been confirmed from 188 countries and regions, resulting in approximately 654,159 people died, and around $9,581,047$ people recovered. While in Indonesia, there were 100,303 confirmed cases of COVID-19, which until now are still increasing, around 4,838 people died, and around 58,173 people recovered. ${ }^{6}$ Clinical trials are ongoing to evaluate potential treatments for COVID-19 due to the absence of vaccines or specific treatment for this disease. ${ }^{2}$ Remdesivir is one of the antiviral drugs that are currently on clinical trials for COVID-19. This drug shows activity against MERS-CoV (Middle East Respiratory Syndrome Coronavirus) and SARS-CoV-1 (Severe Acute Respiratory Syndrome coronavirus 1) in vitro and animal models. ${ }^{7}$ A study shows that remdesivir and chloroquine can inhibit viral infections, but further studies are needed. ${ }^{8}$ A study involving 100 COVID-19 patients with treatment containing chloroquine reported the decrease of pneumonia exacerbation and the reduction of viral load levels. ${ }^{9}$

However, recently, the FDA stated that chloroquine and hydroxychloroquine could cause serious heart rhythm problems in COVID-19 patients who receive this regimen. Chloroquine and hydroxychloroquine are often combined with azithromycin and other drugs that prolong the QT interval in the heart and this can lead to ventricular tachycardia. The FDA recommends that chloroquine and hydroxychloroquine, either alone or in combination with azithromycin, when used for COVID-19 patients, should be limited to clinical trial settings or for treatment in certain hospitals. ${ }^{10}$ Other drugs currently in clinical trials for the treatment of COVID-19 include remdesivir, lopinavir/ritonavir, tocilizumab, and favipiravir. ${ }^{4}$

The best way to prevent and slow the transmission of the SARS-CoV-2 virus is by frequent hand washing and maintaining social distancing between individuals by staying at home and avoiding crowds. ${ }^{2}$ The occurrence of this health emergency will naturally lead to challenges such as quarantine, closure of locations, travel limitations, disruption of the supply chain of medical products, and others. ${ }^{1}$ These challenges can cause difficulties in fulfilling protocols for clinical trial sampling procedures for drug development and therapeutic drug monitoring (TDM), which involve researchers' direct contact with volunteers in the laboratory. It might cause protocol deviations that cannot be avoided because of the COVID-19 pandemic.
An important aspect of clinical trials of drug development and TDM is the accurate quantification of drugs in a given matrix. These aspects include the study of pharmacokinetics, pharmacodynamics, and toxicokinetics to interpret dosages, frequency, and duration of treatment. ${ }^{11}$ The matrices used for sampling are blood, plasma, serum, cerebrospinal fluid, breast milk, urine, saliva, and hair. ${ }^{12}$ Among all alternative sampling, plasma and serum are the most widely used samples for determining the concentration of analytes. Although considered a gold standard, plasma and serum sampling with the venipuncture technique is more invasive and has disadvantages. This sampling requires medical personnel's assistance, storage conditions must be appropriate, and large sample volumes. ${ }^{11}$

Today, efforts to reduce the volume taken in blood sampling have developed by applying microsampling techniques in quantitative bioanalysis. Microsampling technology has been widely used in non-clinical and clinical drug development studies and TDM. Because of the reduction in the sampling volume, patients will be more comfortable, easier to handle, have low shipping costs, and can be stored at room temperature. ${ }^{13,14}$ The majority of microsampling applications for the quantitative determination of analytes have been developed using blood in the form of dried blood samples, namely in Dried Blood Spots (DBS) and Volumetric Absorptive Microsampling (VAMS). Furthermore, to obtain high-quality quantitative bioanalysis data from dried blood samples for the development of this drug has facilitated several high sensitivity and selectivity analysis instrumentation, specifically LC - MS/ MS. $^{13}$

Dried Blood Spots (DBS) have been widely used as an alternative sampling for small and large molecules in various fields such as drug discovery and development, TDM, doping analysis, drug toxicology, and others. However, DBS has disadvantages in the influence of hematocrit (HCT) or volume fraction of red blood cells in blood samples in quantitative tests. HCT plays a vital role in determining blood viscosity so that it can affect the spread of blood samples on DBS paper, which allows the results of biased analysis. ${ }^{15}$

In 2014, the Neoteryx ${ }^{\circledR}$ company launched a new, fixed volume sampling method called Volumetric Absorptive Microsampling (VAMS) ${ }^{15}$ VAMS is used to obtain dried blood specimens and other biological matrices for bioanalysis applications. ${ }^{16}$ The porous hydrophilic tip of VAMS has been designed to absorb fixed sample volume. ${ }^{17}$ Compared to venous sampling, VAMS has several 
advantages, namely, reduction in sampling volume, simple sampling techniques, secure handling, low shipping costs, and can be stored at room temperature. ${ }^{18}$ When compared with DBS, VAMS allows accurate sample volume collection without the influence of HCT which can affect the performance of quantitative methods because it affects sample homogeneity, drying time, analytic recovery, and reproducibility of analysis. ${ }^{19}$ The use of VAMS also does not require medical personnel's assistance so that sampling can be done alone by patients at home. This certainly can slow down the chain of transmission of COVID-19, which requires individuals to maintain social distancing and remain at home.

At present, dry blood microsampling techniques with VAMS are widely used in drug development research and TDM because of easy and self-administered sampling techniques at home. Apart from all the advantages of VAMS compared to another blood sampling, VAMS is still a sampling technique that has not been widely studied because this sampling tool is quite expensive and can only be used for one-time sampling. ${ }^{11}$

Based on the explanation above, the authors did this review to explore the benefits and challenges of using VAMS in drug development studies and TDM during the COVID-19 pandemic. The author will focus on clinical and bioanalytical aspects and potential challenges when applying them and how these challenges can be overcome. This review is expected to make VAMS an alternative consideration for clinical trial sampling in drug development and TDM during the COVID-19 pandemic so that it can be implemented immediately for the sake of ongoing clinical trial protocols for drug development and TDM.

\section{Drug Discovery and Development in General}

Currently, COVID-19 is a new disease that does not yet have a particular drug or vaccine that can cure and prevent the disease. Therefore, a series of suitable drug discovery and development processes are needed to target the disease. ${ }^{20}$ Drug discovery is a process intended to identify small synthetic molecules or large biomolecules for comprehensive evaluation as potential drug candidates. In short, the drug discovery process includes identification of the disease, selection of molecular targets, development of in vitro testing followed by a screening of compounds against targets, and obtaining optimal results for compounds that show potential for biological targets in vitro and in animal model. Furthermore, these compounds are further optimized to improve the efficacy and pharmacokinetic studies before heading for drug development. ${ }^{21}$

The process of drug development can be separated into preclinical and clinical development. In preclinical development, pharmacological, toxicological, and safety studies of candidate drugs are carried out to determine the maximum safe concentration in animals, determine potential side effects, and determine the best formulation. If the candidate shows sufficient efficacy and safety, the drug is permitted by the drug regulatory agencies to begin its clinical development. $^{21}$

In general, the clinical trial process of drug development is divided into several phases. Phase I clinical trials are conducted to assess the drug's safety and tolerability and are usually carried out on 10-100 healthy volunteers. Pharmacokinetic aspects, including ADME (absorption, distribution, metabolism, and elimination) and pharmacodynamics, are also monitored. The maximum tolerated dose is also determined. ${ }^{21}$

Phase II clinical trials investigate the clinical effectiveness of drugs carried out in patients with certain diseases. In this phase, around 50-500 patients receive new drugs that are studied primarily to assess the efficacy of the drug in patients (Sinha \& Vohora, 2017). Drug safety studies have also continued through this phase. In the first part of Phase II, called phase IIA, is to determine the dose needed to provide the desired therapeutic effect. After the exact dosage level is determined, the drug candidate will go through phase IIB where the drug candidate will be determined for its efficacy in a larger population. ${ }^{22}$

Phase III clinical trials confirm the efficacy of the drug being studied in a larger population, usually several hundred to several thousand volunteers/patients. Randomized and multicentric trials (conducted in several places) compare the drugs studied with the best treatment or standard of care for certain diseases. Adverse side effects in patients are also monitored. After obtaining the results, all data to date has been compiled into the drug application file and submitted for approval of drug licensing regulations. After the drug is marketed, post-marketing surveillance or Phase IV clinical trials begin as additional follow-up studies to detect rare or long-term side effects in much larger populations or effects in certain specific populations, drug-drug interactions, and drug-drug interactions disease. Phase IV clinical trials have significant implications, including changing drug labels, contraindications, interactions, and even withdrawal of drugs that are already marketed. ${ }^{21,22}$ 
Please note that clinical trials of drugs are not only applied to new drugs but can be applied to drugs that already exist for specific diseases, and these drugs want to be used for different diseases. Drug clinical trials can also be applied to compare several different treatments for a disease, evaluate biomarkers for a disease, and can be used to determine the cause of a disease or develop knowledge about the mechanism of disease.

\section{Therapeutic Drug Monitoring (TDM) in General}

Therapeutic drug monitoring (TDM) interprets the results of examinations of certain drug levels due to the narrow therapeutic index. ${ }^{23}$ TDM involves measuring the patient's blood or plasma drug levels at the specified time to guide the dosage regimen needed to maintain levels of therapeutic ranges. ${ }^{24}$ By combining pharmaceutical, pharmacokinetics, and pharmacodynamic knowledge, TDM enables the assessment of certain drugs' efficacy and safety. ${ }^{25}$ In particular, TDM provides rational support for the translation of doses to patient individual characteristics and contributes to optimizing the design of a critical phase III clinical trials, where the success of this phase represents the main conditions for drug marketing approval. ${ }^{24}$

The proper use of TDM requires more than just measuring drug concentrations in blood or other matrices and comparing it with the target range. On the contrary, TDM plays a vital role in developing safe and effective therapeutic drugs and the individualization of these drugs. When interpreting drug concentration measurements, factors that need to be considered include the sampling time to the dose, dosage history, patient response, and desired clinical target. This information can be used to identify the most appropriate dosage regimen to achieve an optimal response with minimal toxicity. ${ }^{25}$

Indications for TDM include efficacy, compliance, drug interactions, avoidance of toxicity, and monitoring after therapy discontinuation. The contribution of pharmacokinetic variability to differences in dose requirements can be identified by measuring drug concentrations in blood or other matrices under steady-state conditions and modifying doses to achieve desired concentrations related to effectiveness. ${ }^{24}$

Pharmacological characteristics of drugs that must undergo TDM include, drugs that have a narrow therapeutic index, when there are significant variations in pharmacokinetic parameters between patients, the absence of pharmacodynamic markers of therapeutic response and/or toxicity that is easily assessed for changes in dose, and criticality of the patient's condition for dose adjustment efforts. $^{24}$

\section{Volumetric Absorptive Microsampling (VAMS) at Glance}

VAMS is a sampling method used to obtain dried blood specimens and other biological matrices for bioanalysis. ${ }^{16}$ Compared to venous sampling, the VAMS technique reduces the volume of sampling, the sampling technique is simpler, more comfortable to handle, has low shipping costs, and can be stored at room temperature. The use of VAMS also does not require medical personnel's assistance so that sampling can be done alone at home. ${ }^{18}$

When compared with DBS, the use of the VAMS sampling method enables accurate collection of sample volumes. It reduces the effect of HCT in DBS which can affect the performance of quantitative methods that affect sample bottle formation, homogeneity, drying time, analytic recovery, and durability and reproductive ability of the test. ${ }^{19}$

The VAMS device consists of an absorbent white tip that is attached to a plastic handle. The porous hydrophilic white tip containing cellulose has been designed to absorb a fixed sample volume. VAMS devices are available to collect 10, 20, and $30 \mu \mathrm{L}$ samples and are stored in cartridges ( 2 samplers), clamshells (2-4 samplers), or 96well plates ${ }^{17}$ (depicted in Figure 1).

Sampling is done by dipping the tip surface into the patient's blood from a finger or heel prick at a $45^{\circ}$ angle. The tip of the sampler will absorb blood in a few seconds. The tip of the sampler should not be submerged in the blood until it passes over the shoulder of the tip, because it can lead to trapping excess blood on the plastic handle. After sampling, VAMS is dried for 2 hours at room temperature before it is sent or stored before analysis. When preparing sample analysis, the tip of the sampler containing the sample is used for the extraction process. ${ }^{17}$

When extracting analytes, the tip of the VAMS is usually (not always) removed from the plastic handle and inserted into a microtube or microtiter plate. The extraction process can be divided into two different stages, namely, desorption and analyte extraction. These steps can be carried out simultaneously with a single solvent or sequentially using additional extraction procedures. The solvent commonly used in the extraction of VAMS is 


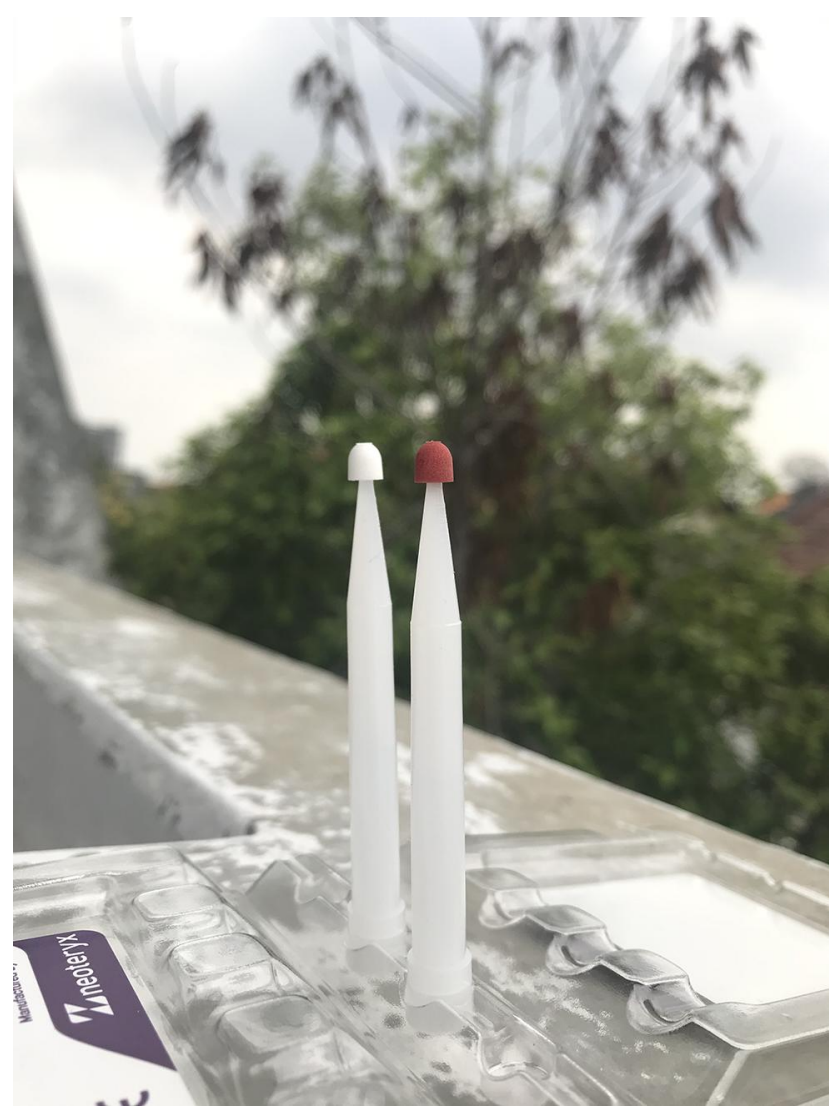

Figure I The VAMS Mitra ${ }^{\circledR}$ cartridge (2 samplers).

methanol or water (Protti et al, 2019). In addition, the preparation of VAMS samples is also easy because it only needs to separate the VAMS tip with the handle without having to cut out like a sub-punch method that must be applied to DBS samples. ${ }^{11}$

Thus, VAMS has the advantage in sample preparation, where sample preparation becomes simpler and does not need to go through the centrifugation stage before extracting it. However, VAMS has a disadvantage that the sample can only be processed once, so a lot of sampling must be done if the analysis is done more than once, and this sampling tool is also quite expensive compared to DBS. ${ }^{17}$

\section{Clinical Considerations for Implementation of VAMS}

This section will focus on the considerations in the implementation of VAMS technology in clinical programs. Simultaneously, the discussion for the use of VAMS in bioanalysis will be detailed later in this manuscript. Several published studies have discussed recommendations for DBS implementation in clinical programs. ${ }^{26-28}$
The authors consider that the use of VAMS has more advantages when used in clinical programs compared to DBS, and the analysis will be explained in this section. Therefore, workflows using VAMS are recommended and should be considered by those who apply this technology in clinical trials and TDM during the COVID-19 pandemic.

\section{Clinical Considerations for} Implementation of VAMS to Clinical Trials and TDM During the COVID-I 9

\section{Pandemic}

VAMS technology has many advantages when applied as a sampling tool in clinical trials. In particular, invasive nature is minimal when sampling and the volume of blood taken is small. This makes the VAMS technique an attractive approach for vulnerable populations, such as neonates or pediatrics and the elderly. In addition, blood sampling only from the fingertips allows this technique to be done alone at home without medical personnel's help. This advantage can be applied to drugs that target acute episodic disease. In this condition, researchers are required to collect samples that cannot be obtained through conventional sampling, for example, in children with epilepsy and patients with migraines. ${ }^{29}$ Numerous publications have supported the use of VAMS in clinical trials. A list is provided in Table 1.

For analytes that have optimum stability in dry samples at room temperature, the use of VAMS is very advantageous for clinical trials conducted in remote areas with limited access to centrifugation devices, freezers, and frozen sample transfers. This is because VAMS does not have to use a centrifuge to separate analytes, and sample shipments and sample storage do not require a freezer like intact blood samples. Also, when compared to DBS, the VAMS sample enables easy sample preparation because it only needs to separate the VAMS tip from the handle without having to cut like a sub-punch method (cutting DBS samples with a consistent diameter) which must be applied to DBS samples. ${ }^{11}$

In some cases of blood sampling through venipuncture, which is also followed by the addition of anticoagulants in clinical trials, it is necessary to collect large numbers of samples at several time points to determine the absorption, distribution, metabolism, and elimination parameters in pharmacokinetic studies. Therefore, blood sampling via finger prick is preferred because it does not require anticoagulants, 


\begin{tabular}{|c|c|c|c|c|c|c|c|c|c|c|c|c|c|}
\hline 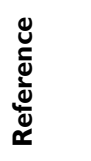 & q & ఫ & in & $\bar{n}$ & in & in & t. & 号 & $\underline{\infty}$ & i̊ & in & $\stackrel{\infty}{n}$ & $\underline{\underline{n}}$ \\
\hline 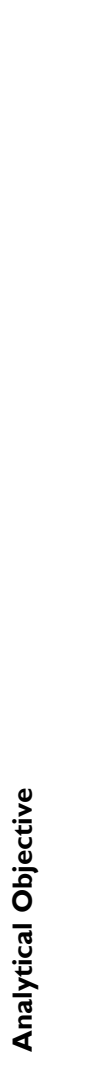 & 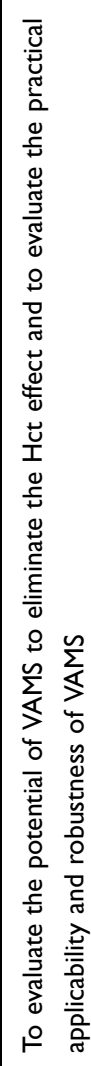 & 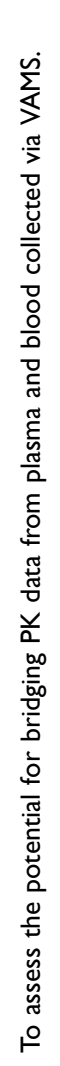 & 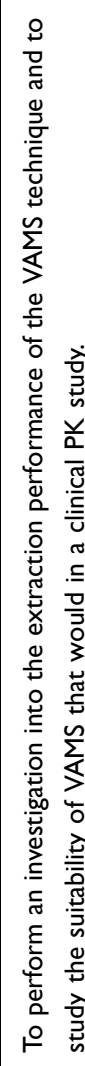 & 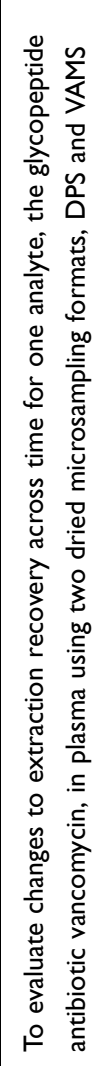 & 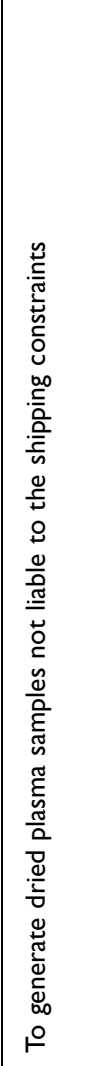 & 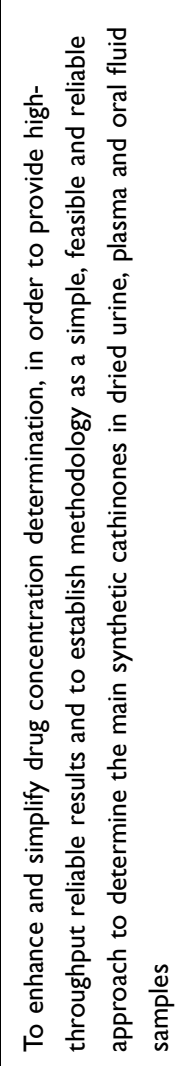 & 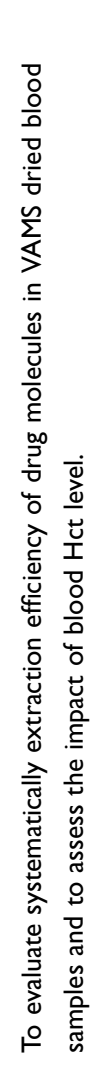 & 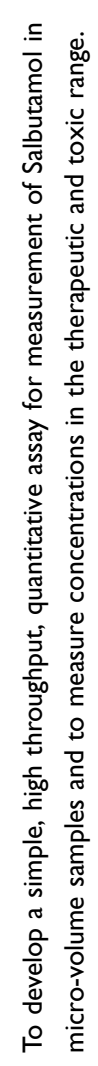 & 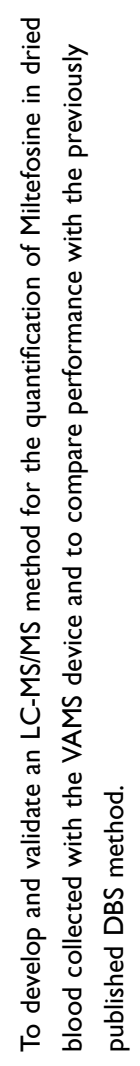 & 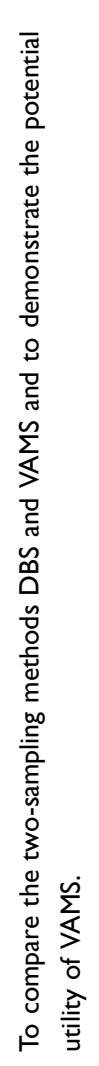 & 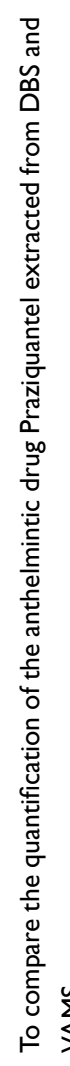 & 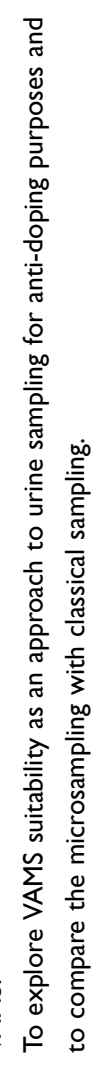 & 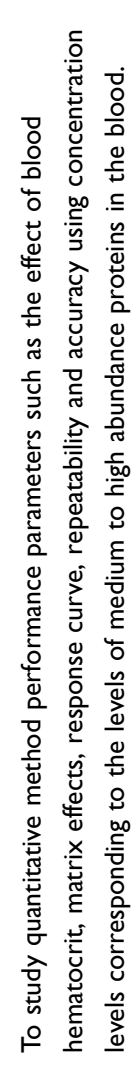 \\
\hline 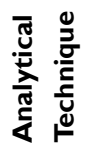 & 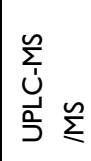 & 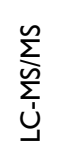 & 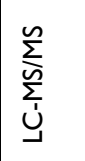 & $\sum_{j}^{\infty}$ & 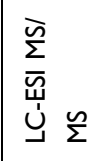 & $\sum_{\substack{n \\
\vdots}}^{n}$ & 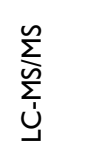 & $\sum_{U}^{n}$ & $\sum_{U}^{\infty}$ & 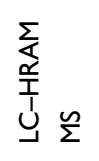 & 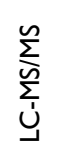 & 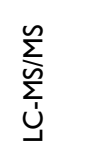 & 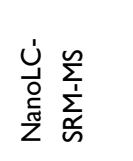 \\
\hline 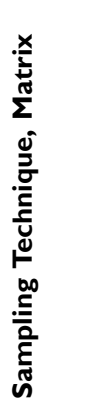 & 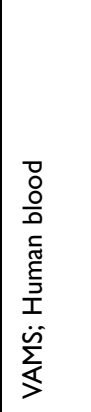 & 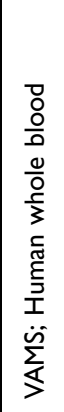 & 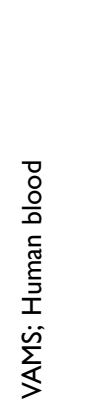 & 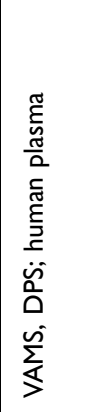 & 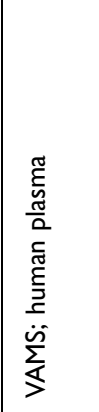 & 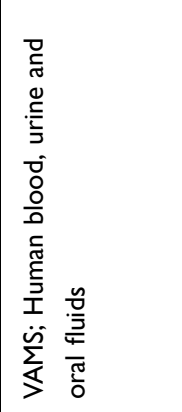 & 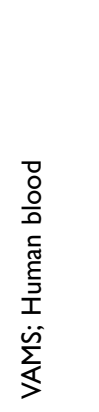 & 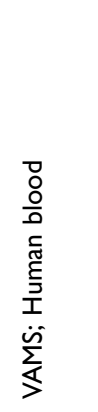 & 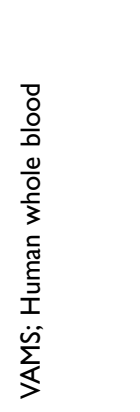 & 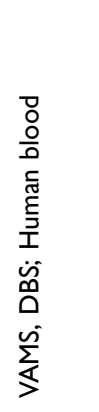 & 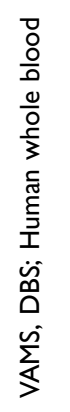 & 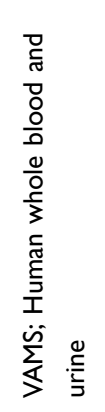 & 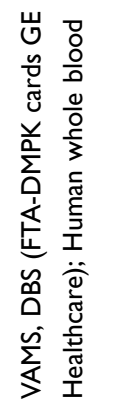 \\
\hline 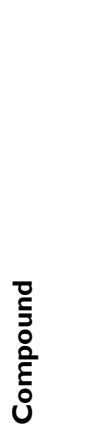 & 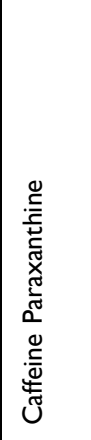 & 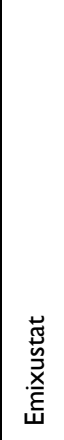 & 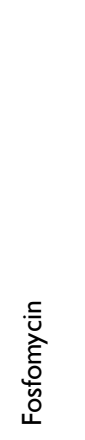 & 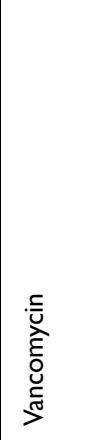 & 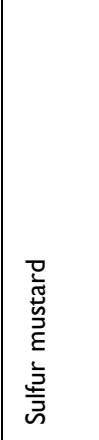 & 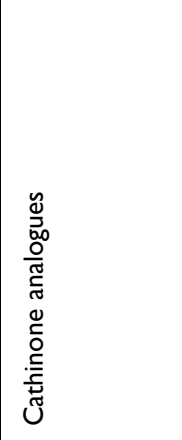 & 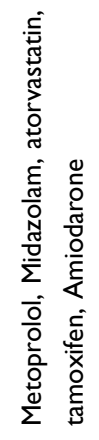 & 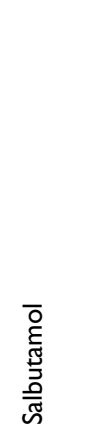 & 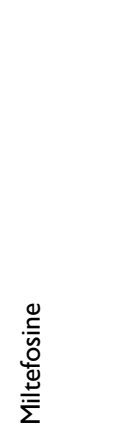 & 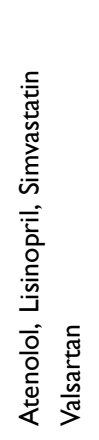 & 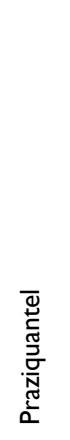 & $\begin{array}{l}\text { סे } \\
\text { ơ } \\
\text { X̀ } \\
0\end{array}$ & 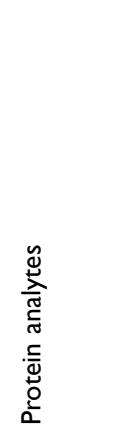 \\
\hline
\end{tabular}




\begin{tabular}{|c|c|c|c|}
\hline in & : & $\overline{0}$ & $\widetilde{\sigma}$ \\
\hline 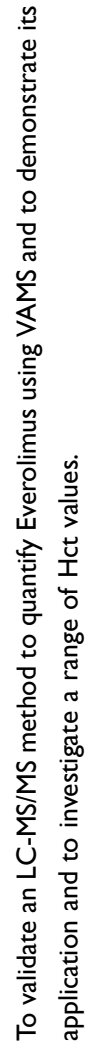 & 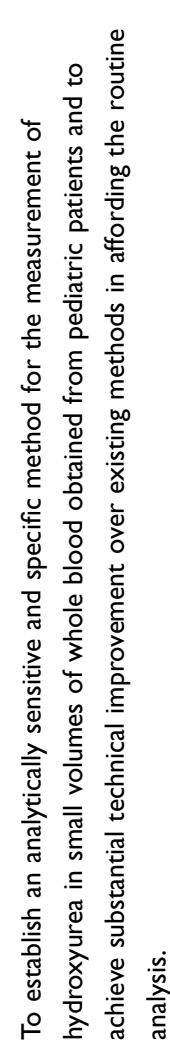 & 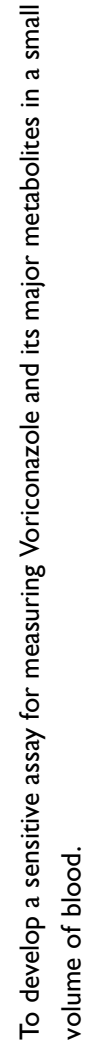 & 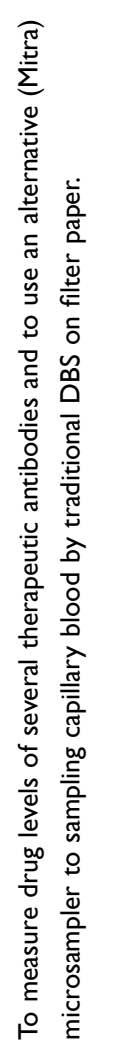 \\
\hline 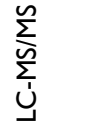 & 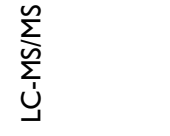 & $\sum_{-}^{\infty}$ & 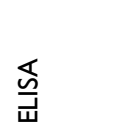 \\
\hline 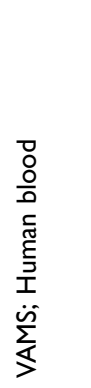 & 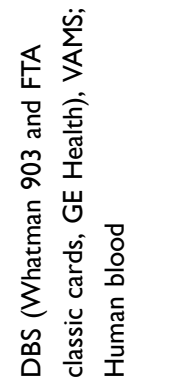 & 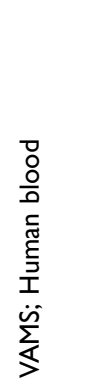 & 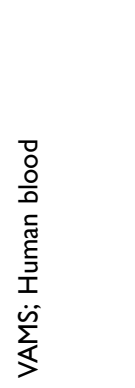 \\
\hline 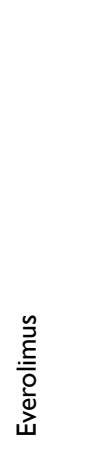 & 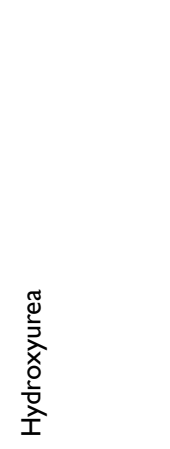 & 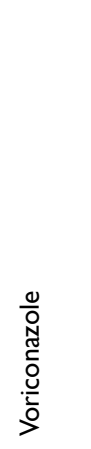 & 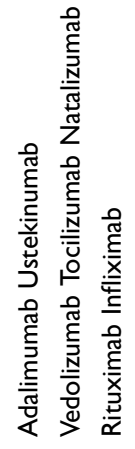 \\
\hline
\end{tabular}

centrifugation devices, and blood samples taken are also small. ${ }^{13}$ This can increase the comfort of volunteers/patients if sampling for pharmacokinetic studies in clinical trials and TDM requires large samples from several time points of collection.

In addition to the benefits of patient comfort, the VAMS approach can also save on the costs of storing and sending samples. This is reviewed from the use of sample storage at room temperature and dry sample delivery. Patients' ability to samples at home can also save high costs of travel costs and time associated with patients not having to go to a central clinic. When connected with the current pandemic situation which requires people to stay at home and maintain social distancing, VAMS can be applied immediately for the sampling of volunteers/clinical trial patients at home for the continuation of clinical trials on drug development and TDM.

Unfortunately, the cost-effectiveness of this VAMS can only be applied to the cost of storage, sample delivery, and patient travel costs. One of the disadvantages of VAMS in terms of cost is that this sampling tool is quite expensive and can only be used once. Thus, the handling and preparation of samples required specialized expertise from the analyst in processing the sample to get maximum results and save costs. In addition, because VAMS samples can only be used and processed once, sampling is needed more than once so that if there are errors in testing, there are still backup samples that can be processed.

\section{Clinical Considerations for Implementation of VAMS to Clinical Trials and TDM for COVID-19}

The FDA issued guidance focusing on implementing drug development for COVID-19 during this pandemic. The FDA states that in clinical trials the development of drugs for the treatment and prevention of COVID-19 must involve high-risk patients such as elderly people who have complications, pediatrics or children, people with cardiovascular or respiratory diseases, diabetes, chronic kidney disease, and other comorbidities. ${ }^{30}$ These patients are vulnerable, and their blood may have been taken several times for routine checking by doctors. Therefore, if the researcher involves these patients for clinical trials developing drugs and TDM for COVID-19 disease, they should be treated as minimally as possible. As previously explained, VAMS allows for the sampling of vulnerable patients because of the small sampling and 
minimally invasive nature that can improve patient comfort.

So far, there are still no drugs or vaccines that have been declared effective in curing and preventing COVID19. However, as previously explained that several antiviral drugs are in the process of research by researchers to get FDA approval. Drugs currently in clinical trials for the treatment of COVID-19 include remdesivir, lopinavir/ritonavir, tocilizumab, favipiravir, and chloroquine. ${ }^{4}$ These drugs have previously been indicated for certain diseases, such as remdesivir, which is effective compared to lopinavir/ritonavir in the urine model of patients with MERS$\mathrm{CoV}$ infection. ${ }^{31}$ However, for the use of remdesivir and other drugs as COVID-19 treatment regimens, they must first go through clinical trials to test safety, see the drug's pharmacokinetics, and the effectiveness of the drug to make it a COVID-19 treatment.

Chloroquine, a recommended drug that is often used to cure COVID-19 patients, is reported to cause heart side effects at concentrations above the maximum plasma concentration. Chloroquine plasma concentration after the first dose is given ranges $65-226 \mathrm{ng} / \mathrm{mL}$. Chloroquine TDM is recommended to determine the dosage regimen required for each patient receiving chloroquine and ensure plasma concentrations remain in the therapeutic range to maximize effectiveness and minimize toxic effects. ${ }^{32}$ Therefore, drug monitoring is a rate-limiting step for drug development and could speed up drug development in order to have an effective COVID-19 treatment knowing that every day the number of COVID-19 patients continues to grow.

In carrying out clinical trials of drug development and TDM for the treatment of COVID-19, careful consideration is needed regarding the cost-effectiveness and ease of handling samples because this test may last for several years. To accomplish this, alternative VAMS sampling tools can be used to reduce research costs because vials, sample cups, and other sample preparation tools are used less because the sample volume is small, does not require anticoagulants, does not require centrifugation tools for analytic extraction, does not require a freezer for storage and samples can be sent in dry samples at room temperature.

At present, the NIH (National Institute of Health) in the United States is conducting studies to measure asymptomatic COVID-19 infection cases using the VAMS approach. The NIH attempted to study adults in the United States who had no history of infection with SARS$\mathrm{CoV}-2$ but had antibodies to the virus. This study analyzes blood samples from 10,000 volunteers to provide essential data for the epidemiological study model. The volunteers who participated in this study will conduct sampling at home using VAMS. The researchers stated that the use of VAMS in this study could facilitate sampling and continuity of research because participants can take their samples at home. The researchers also stated that they have experience using VAMS to track the spread of other infectious diseases such as influenza. This method is safe, effective, and easy to use. This study, which only involves finger prick sampling from participants, is expected to help researchers fight COVID-19 from home. ${ }^{33}$

\section{Factors to be Considered in the Use of VAMS for Clinical Trials and TDM During the COVID-19 Pandemic}

The quality of samples collected by microsampling, including VAMS, is crucial for the study's success. Therefore, it is also important to conduct training on sampling using VAMS, especially if the sample collection is done by volunteers/patients at home without medical personnel's help. Self-sampling without medical staff or experts help allows results with more significant data variability. ${ }^{34}$

Although the use of VAMS is fast, easy, and possible to absorb a fixed volume, its application still has factors that can affect variations in sample volume if it does not follow the established procedures. In VAMS sampling, the tip of the sampler should not be immersed in blood until it passes over the tip of the shoulder, because it can result in trapping excessive blood on the plastic handle. ${ }^{17}$ This must be confirmed and conveyed to volunteers/patients who will take their own sample at home to avoid variability in results.

Efforts that can be made are using some media to socialize and train volunteers/patients to take samples with VAMS correctly. This can be considered, given the quarantine and social distancing situation during the COVID-19 pandemic, which requires people to remain at home and cannot be monitored and controlled directly by the responsible medical personnel/researcher. Medias that can be used include online training forums, video tutorials, and instruction brochures. These efforts have been proven to increase the acquisition of high-quality samples. ${ }^{35}$ Training efforts with the media must ensure that study volunteers/patients fully understand the sampling technique and produce reliable results. 
Another factor that can influence the success of the analysis with the VAMS sampling tool is volunteers/ patients' compliance in taking samples, especially if done alone at home without strict control. Before volunteers/ patients participate in clinical trials, volunteers/patients are required to read and sign an informed consent that contains the purpose of the study and why the volunteers/ patients are asked to participate, research procedures and what actions will be carried out, research benefits, risks and possible side effects, contact information, statements that participation in research is voluntary, and participants may withdraw if they wish, and consent sheets for participation in research. ${ }^{36}$ By signing the informed consent, the volunteers/patients are considered to have understood the objectives, risks, and procedures of the study and agreed to participate voluntarily in the study and can comply with the rules to get the maximum research results set by the sponsor/researcher.

Despite having signed the informed consent, the discipline of volunteers/patients in sampling with VAMS at home without direct control remains a significant concern. Mainly if VAMS is applied for use in Indonesian people who have low discipline during the COVID-19 pandemic. Commitment to discipline in involvement in clinical studies comes from each person, and it must be balanced with regulations and policies that have been made by the sponsor/researcher. Efforts that might be implemented to reduce voluntary/patient indiscipline in-home sampling with VAMS are to carry out periodic control via telephone contact or online forums and send reminders with short messages about sampling schedules and sample delivery.

\section{Bioanalytical Considerations for Implementation of VAMS}

Bioanalysis has an important role in the process of drug development. At present, bioanalysis is an essential part of evaluating drug toxicity and pharmacokinetics and pharmacodynamics of drugs. Bioanalysis involves identifying and quantifying analytes in biological samples such as blood, plasma, serum, saliva, urine, feces, skin, hair, and organ tissues. Bioanalysis is also carried out in clinical, preclinical, and forensic toxicology laboratories. Thus, bioanalysis is essential in many fields of research, such as the development of new drugs, forensic analysis, doping control, and identification of biomarkers for the diagnosis of many diseases. Liquid chromatography instruments combined with mass spectrometry (MS/MS) tandem have been used in drug bioanalysis. Bioanalysis procedures include sampling, sample preparation, analysis, calibration, evaluation, and data reporting. ${ }^{37}$

In studies using microsampling, method validations are needed to ensure the sensitivity and selectivity of the analysis and provide reliable quantitative data results because the sample volume used is small. Therefore, method validations for workflows using microsampling must also include accuracy, precision, selectivity, sensitivity, recovery, stability, carryover, and matrix effects. ${ }^{38,39}$ This validation must overcome, at a minimum, the effects of storage and handling temperatures, homogeneity of sampling, HCT, and stability. In this section, the authors discuss the analysis considerations when using VAMS and the challenges that might be discovered.

\section{Bioanalytical Considerations for Implementation of VAMS in Clinical Trials and TDM}

Fixed Volume Uptake

A fixed sample volume is key to the accuracy of analysis in quantitative bioanalytic research. For conventional sampling, blood collection requires high accuracy. After the samples are collected, the samples will be processed, frozen, and then sent to a bioanalysis laboratory. All is done by analytical chemists who are trained using calibrated equipment, either manual pipettes or automated liquid handlers in the bioanalysis laboratory. ${ }^{40}$ In the use of DBS, each blood spot's diameter is a more bigger concern than the volume of blood. Different types of DBS papers require different amounts of blood to produce identical blood spot diameters. ${ }^{11}$

To overcome the problem of consistent sample volume, the use of VAMS allows the collection of fixed sample volumes starting from 10,20 , and $30 \mu \mathrm{L} .{ }^{16}$ Sampling can be carried out by personnel in health facilities, clinics, or even patients at home because of the ease of use. However, VAMS sampling must be monitored because errors can occur if the collection method is not appropriate. As explained earlier, VAMS sampling should not exceed the device handle's shoulder as this can cause excess sampling volume. Therefore, it is crucial to monitor the correct use of VAMS in sampling even though the device itself allows it to be used by personnel who are not experts in analytical or clinical fields. Training through adequate media can educate operators or users about the use and handling of appropriate devices to reduce errors. ${ }^{41}$ Also, if direct training 
is not possible in this pandemic situation, the operator or user can watch video instructions or brochures on the appropriate usage methods that are already available.

\section{Stability of Analytes and Metabolites}

A critical parameter for microsampling evaluation is stability. This is to ensure sample integrity and data consistency during long-term studies in a series of bioanalytic studies. The analyte concentration at the time of analysis after delivery must represent the concentration collected at the time of sampling. ${ }^{42}$ Storage and sample delivery contribute significantly to analytes degradation. Temperature, humidity, and sunlight can also cause degradation of analytes in dried blood samples. ${ }^{11}$

The instability of analytes and metabolites can also be caused by chemical reactions or degradation catalyzed by enzymes. When sampling with conventional methods, degradation can be minimized by adding enzyme inhibitors, controlling the sample's $\mathrm{pH}$, and antioxidants to the collection tubes before sampling. ${ }^{43}$ Whereas in the use of microsampling, such as VAMS and DBS, the addition of these components is more often added during extraction. This can allow the degradation of analytes that are less stable when drying samples that take several hours.

Several studies have been conducted to test the stability of various analytes in VAMS samples. Stability is examined under different storage conditions, ranging from $-80^{\circ}$ $\mathrm{C}$ to $50^{\circ} \mathrm{C}$ at the specified time. In several studies, the stability of analytes in VAMS samples produced different results depending on the analytes studied. ${ }^{17}$

One study examining the drug miltefosine found that the drug was stable in VAMS samples for at least one month at room temperature $\left(22 \pm 3^{\circ} \mathrm{C}\right)$, whereas in DBS, it could last for five months at room temperature in an aluminum bag. This can be a worrying issue in the use of VAMS. However, it should also be noted that the storage conditions of the two samples are slightly different because the VAMS samples are stored in the dark in a clamshell storage container and the DBS samples are stored in a closed aluminum bag with three desiccant bags which allows different results in the stability of the analyte in different samples. ${ }^{18}$

Testing the stability of analytes can also be seen in studies with emixustat drugs for eye diseases. Samples spiked by emixustat are absorbed with VAMS and left at room temperature $\left(25^{\circ} \mathrm{C}\right)$, refrigerators $\left(4^{\circ} \mathrm{C}\right)$, and freezer $\left(-20^{\circ} \mathrm{C}\right)$ for up to three months before extraction. Samples were kept at stable room temperature for about one week with a maximum bias of $12 \%$. Samples were then processed after three months of storage at room temperature and showed a reduction in recovery of up to $35 \%$. Emixustat stability in VAMS increases at lower storage temperatures where the sample is stable for one month in the refrigerator and stable for three months in the freezer. ${ }^{44}$

In general, the stability of analytes in VAMS samples depends on the type of the studied analyte. If the analyte is stable at room temperature, shipping and storage costs can be significantly reduced because there is no need to freeze it. However, the stability of other analytes cannot be ensured for a long time at room temperature. The analyte's stability can be improved by storing the sample at lower temperatures before or after the extraction of the analyte.

\section{Homogeneity of Sample and Hematocrit Effects (HCT)}

Several research papers on the problem of blood HCT levels and the homogeneity of sample dispersion in the use of DBS that have led to biased results have been widely documented. In short, blood with different HCT values will affect the blood viscosity, which leads to variations in the size of the sample distribution on DBS paper, which can make the analysis results biased. To overcome this problem, VAMS technology is designed to ensure proper blood volume collection at all times of sampling with minimal HCT effects. ${ }^{45}$ This is evidenced by protein studies using VAMS, where the observed results have no visible relationship between signal intensity with different HCT concentrations in proteins. ${ }^{15}$ Also, high HCT can reduce recovery from analytes. When HCT concentrations are high, high red blood cell counts can be trapped on DBS paper substrates, which can inhibit the analytic desorption process. ${ }^{46}$ This was proven in the study of the drug miltefosine, which had a higher recovery value using VAMS compared to DBS. $^{18}$

In addition, studies have also been documented using human blood and mice with different HCT absorbed by VAMS. The average volume of human and rat blood collected was in the estimated range of HCT $20-65 \%$. The results of the blood volume slope to the HCT plot are close to zero, and this shows that the volume of blood collected by VAMS has a slight variation with different blood $\mathrm{HCT}$ values. ${ }^{47}$

\section{Carryover and Cross-Contamination}

Carryover and cross-contamination during sample collection can cause significant experimental errors in the analysis process because they can influence the selectivity of the analysis. ${ }^{13}$ Therefore, collection and handling procedures and storage 
technology must be designed to eliminate or minimize accumulation and contamination. DBS's use allows for contamination due to the design of DBS, which has no protection for long periods of drying. To deal with this, VAMS technology is equipped with a cartridge protector that allows drying of samples in closed conditions. This can minimize contamination from the environment, which can affect the results of the analysis.

\section{Logistics}

As explained earlier, VAMS can provide practical benefits. For example, dry sampling with VAMS lowers costs, and the sampling process is more manageable by allowing samples to be stored and sent at room temperature. VAMS is designed to collect samples at home and be equipped with collection devices and shipping containers into one cartridge. This approach makes it easy for users to use it, and sending samples to the bioanalysis laboratory becomes cheaper because it can be done at room temperature.

\section{Bioanalytical Considerations for Implementation of VAMS in Sample Analysis \\ Accuracy and Precision}

In the series of bioanalytic studies, the analytical method used must be specific to the analyte to be measured and must demonstrate pre-determined accuracy and precision. Testing the accuracy and precision during the development of a method is essential to determine whether this method is suitable for analyzing research samples. The acceptance criteria for accuracy and precision are at an average concentration of $\% \mathrm{CV}$ not more than $\pm 15 \%$ for QC samples and no more than $\pm 20 \%$ for the LLOQ area.

In studies examining proteins with VAMS, it is evident that the results of the accuracy and precision of the analysis obtained met the requirements of accuracy and precision criteria. ${ }^{15}$ Compared to DBS, the research of miltefosine with VAMS sampling method has an accuracy bias that meets the requirements of accuracy and precision criteria. At the same time, the accuracy and precision of DBS samples do not meet the requirements. These results prove that the VAMS sample can improve the accuracy and precision of the analysis compared to the DBS sample. ${ }^{18}$

\section{Recovery and Matrix Effects}

The recovery of the sample and the ability to extract must be optimized for the microsampling approach to ensure test performance and maximize test sensitivity. As previously explained, VAMS technology makes it possible to increase recovery from analytes because it can minimize the effects of HCT, especially on higher HCT. In all cases, recovery from analytes must be optimized. One way to optimize recovery values is to choose the appropriate extraction solution and optimize the sonication time in sample preparation.

The use of microsampling, such as VAMS and DBS, tends to have fewer matrix effects than conventional sampling. This is because the lower sample volume and sample processing for attracting analytes from the matrix has more steps than conventional sampling. ${ }^{13}$

\section{Future Prospective}

Based on the literature research and analysis, the authors consider that developments in the use of VAMS for clinical trials of drug development and TDM during the COVID-19 pandemic will be very useful because of the many practical benefits which include easy delivery and storage of samples, and also the ability to collect samples with minimally invasive treatment compared to the conventional venipuncture techniques. A few publications have supported the concordance between the data obtained by two different techniques, VAMS vs wet samples in human. A list is provided in Table 2.

VAMS will also facilitate the collection of samples in locations far from the clinic or can be done at home. This will be more convenient for volunteers/patients, result in simpler clinical trial design, and increase subject recruitment and retention. However, in practice, the use of VAMS has concerns about the discipline of volunteers/patients in collecting samples at home that are not strictly controlled by the sponsor/researcher during the COVID-19 pandemic. To reduce the indiscipline, several media can be used, such as training videos or instruction brochures to socialize and train volunteers/patients on how to take samples with VAMS correctly. Remote monitoring through telephone contacts, online forums, or reminders can also be applied for periodic monitoring. This effort is expected to be implemented if VAMS is applied to Indonesian people who have low discipline.

When viewed from the aspect of bioanalysis, VAMS proves to increase accuracy, precision, and recovery analysis. Despite concerns about the stability of analytes in VAMS samples, this technology is still considered a smart and modern breakthrough in clinical and bioanalysis fields. Therefore, the authors suggest that if an outbreak occurs that requires the holding of quarantine again or an event that does not allow researchers to meet with the subject, 


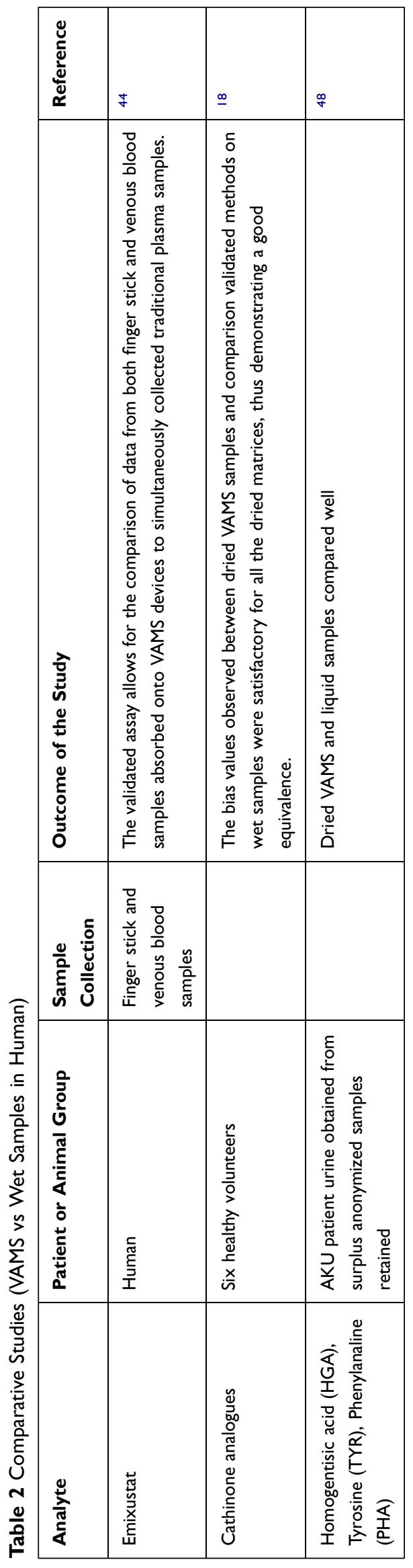

this VAMS technology can be applied to continue clinical trial protocol for drug development and TDM.

\section{Conclusions}

Based on the analysis above, the author can conclude the following things:

- The use of VAMS as an alternative sampling allows the collection of samples in locations far from the central clinic and can be done by the patient at home. This can be applied to the implementation of clinical trials on drug development and TDM during the COVID-19 pandemic, which required quarantine. However, remote monitoring to monitor voluntary/ patient discipline in sampling must be carried out for the success of the study.

- In the aspect of bioanalysis, VAMS has the advantage of minimally invasive treatment and a small sampling volume compared to conventional sampling methods. In addition, VAMS can improve the accuracy, precision, and recovery of the analysis by minimizing the effects of HCT resulting from DBS's use. However, despite its advantages, VAMS has concerns about the stability of analytes that are not stable in dry samples. So special attention is needed on the stability of these analytes in VAMS samples.

\section{Abbreviations}

$\% \mathrm{CV}$, coefficient of variation (\%); ADME, absorption, distribution, metabolism, and elimination; COVID-19, coronavirus disease 2019; DBS, dried blood spots; FDA, U.S Food and Drug Administration; HCT, hematocrit; LC-MS /MS, liquid chromatography with tandem mass spectrometry; LLOQ, lower limit of quantification; MERS-COV, Middle East respiratory syndrome coronavirus; NIH, National Institute of Health; SARS-COV-1, severe acute respiratory syndrome coronavirus 1 ; SARS-COV-2, severe acute respiratory syndrome coronavirus 2; TDM, therapeutic drug monitoring; VAMS, volumetric absorptive microsampling; WHO, World Health Organization.

\section{Highlights}

- Clinical considerations and factors to be considered in the use of VAMS in clinical trials and TDM during the COVID-19 pandemic

- Bioanalytical considerations and recommendations in the use of VAMS as a sampling alternative 
- Challenges to solve and advantages in the use of VAMS as a sampling alternative in clinical trials and TDM during the COVID-19 pandemic

\section{Disclosure}

The authors report no conflicts of interest in this work.

\section{References}

1. FDA. FDA Guidance on Conduct of Clinical Trials of Medical Products during COVID-19 Pandemic Guidance for Industry, Investigators, and Institutional Review Boards. 2020; Available from: https://www.fda.gov/regulatory-information/search.

2. World Health Organization. y. 2020. Available from: https://www. who.int/health-topics/coronavirus\#tab=tab_1. Accessed March 7, 2020

3. CDC. What you should know about COVID-19 to protect yourself and others. Coronavirus Dis. 2020;314937.

4. Chavez S, Long B, Koyfman A, Liang SY. Coronavirus Disease (COVID-19): a primer for emergency physicians. Am J Emerg Med. 2020. doi:10.1016/j.ajem.2020.03.036

5. Van Doremalen N, Bushmaker T, Morris DH, Holbrook MG, Gamble A, Williamson BN. Aerosol and surface stability of SARS-CoV-2 as compared with SARS-CoV-1. $N$ Engl $J$ Med. 2020;382(16):1564-1567. doi:10.1056/NEJMc2004973

6. JHU. Coronavirus COVID-19 Global Cases. 2020. Available from: https://coronavirus.jhu.edu/map.html. Accessed December 16, 2020.

7. Gordon C, Thesnokov E, Feng J, Porter D, Gotte M. The antiviral compound remdesivir potently inhibits RNA-dependent RNA polymerase from Middle East respiratory syndrome coronavirus. J Biol Chem. 2020;295(15):4773-4779. doi:10.1074/jbc.AC120.013056

8. Wang M, Cao R, Zhang L, et al. Remdesivir and chloroquine effectively inhibit the recently emerged novel coronavirus $(2019-\mathrm{nCoV})$ in vitro. Cell Res. 2020;30(3):269-271. doi:10.1038/s41422-020-0282-0

9. Gao J, Tian Z, Yang X. Breakthrough: chloroquine phosphate has shown apparent efficacy in treatment of COVID-19 associated pneumonia in clinical studies. Biosci Trends. 2020;14(1):72-73. doi: $10.5582 /$ bst. 2020.01047

10. FDA. FDA cautions against use of hydroxychloroquine or chloroquine for COVID-19 outside of the hospital setting or a clinical trial due to risk of heart rhythm problems [Internet]. 2020. Available from: https://www.fda.gov/drugs/drug-safety-and-availability/fda-cautionsagainst-use-hydroxychloroquine-or-chloroquine-covid-19-outsidehospital-setting-or. Accessed March 13, 2020

11. Londhe V, Rajadhyaksha M. Opportunities and obstacles for microsampling techniques in bioanalysis: special focus on DBS and VAMS. J Pharm Biomed Anal. 2020;182:113102. doi:10.1016/j. jpba.2020.113102

12. Avataneo V, D'Avolio A, Cusato J, Cantù M, De Nicolò A. LC-MS application for therapeutic drug monitoring in alternative matrices. J Pharm Biomed Anal. 2019;166:40-51. doi:10.1016/j.jpba.2018.12.040

13. Spooner N, Anderson KD, Siple J, Wickremsinhe ER, Xu Y, Lee M. Microsampling: considerations for its use in pharmaceutical drug discovery and development. Bioanalysis. 2019;11(10):1015-1038. doi:10.4155/bio-2019-0041.

14. Martial LC, Aarnoutse RE, Schreuder MF, Henriet SS, Brüggemann RJM, Joor MA. Cost evaluation of dried blood spot home sampling as compared to conventional sampling for therapeutic drug monitoring in children. PLoS One. 2016;11(12):e0167433. doi:10.1371/journal.pone. 0167433 .

15. Andersen IKL, Rosting C, Gjelstad A, Halvorsen TG. Volumetric absorptive MicroSampling vs. other blood sampling materials in LCMS-based protein analysis - preliminary investigations. $J$ Pharm Biomed Anal. 2018;156:239-246. doi:10.1016/j.jpba.2018.04.036
16. Protti M, Mandrioli R, Mercolini L. Tutorial: volumetric absorptive microsampling (VAMS). Anal Chim Acta. 2019;1046:32-47. doi:10.1016/j.aca.2018.09.004

17. Kok MGM, Fillet M. Volumetric absorptive microsampling: current advances and applications. $J$ Pharm Biomed Anal. 2018;147:288-296. doi:10.1016/j.jpba.2017.07.029

18. Kip AE, Kiers KC, Rosing H, Schellens JHM, Beijnen JH, Dorlo TPC. Volumetric absorptive microsampling (VAMS) as an alternative to conventional dried blood spots in the quantification of miltefosine in dried blood samples. J Pharm Biomed Anal. 2017;135:160-166. doi:10.1016/j.jpba.2016.12.012

19. Barco S, Castagnola E, Moscatelli A, Rudge J, Tripodi G, Cangemi G. Volumetric adsorptive microsampling-liquid chromatography tandem mass spectrometry assay for the simultaneous quantification of four antibiotics in human blood: method development, validation and comparison with dried blood spot. $J$ Pharm Biomed Anal. 2017;145:704-710. doi:10.1016/j.jpba.2017.07.033

20. Nikhat S, Fazil M. Overview of Covid-19; its prevention and management in the light of Unani medicine. Sci Total Environ. 2020;728:138859. doi:10.1016/j.scitotenv.2020.138859

21. Sinha S, Vohora D. Drug Discovery and Development: An Overview, In: Pharmaceutical Medicine and Translational Clinical Research. Jamia Hamdard University, New Delhi, India. 2017: 19-32. doi:10.1016/B978-0-12-802103-3.00002-X

22. Meinert CL. Clinical Trials: Design, Conduct, and Analysis. New York: Oxford Univ Press; 2012.

23. Menteri Kesehatan Republik Indonesia. Peraturan Menteri Kesehatan Republik Indonesia Nomor 72 Tahun 2016 Tentang Standar Pelayanan. Kefarmasian Di Rumah Sakit. 2016;8(3):6-10.

24. Buclin T, Thoma Y, Widmer N, et al. The steps to therapeutic drug monitoring: a structured approach illustrated with imatinib. Front Pharmacol. 2020;11(March):1-10. doi:10.3389/fphar.2020.00177

25. Kang JS, Lee MH. Overview of therapeutic drug monitoring. Korean J Intern Med. 2009;24(1):1-10. doi:10.3904/kjim.2009.24.1.1

26. Xu Y, Woolf EJ, Agrawal NGB, Kothare P, Pucci V, Bateman KP. Merck's perspective on the implementation of dried blood spot technology in clinical drug development - why, when and how. Bioanalysis. 2013;5(3):341-350. doi:10.4155/bio.12.321

27. Evans C, Arnold M, Bryan P, et al. Implementing dried blood spot sampling for clinical pharmacokinetic determinations: considerations from the IQ Consortium Microsampling Working Group. AAPS J. 2015;17(2):292-300. doi:10.1208/s12248-014-9695-3

28. Kothare PA, Bateman KP, Dockendorf M, et al. An integrated strategy for implementation of dried blood spots in clinical development programs. AAPS J. 2016;18(2):519-527. doi:10.1208/s12248-015-9860-3

29. Linder C, Wide K, Walander M, Beck O, Gustafsson LL, Pohanka A. Comparison between dried blood spot and plasma sampling for therapeutic drug monitoring of antiepileptic drugs in children with epilepsy: A step towards home sampling. Clin Biochem. 2017;50 (7-8):418-424. doi:10.1016/j.clinbiochem.2016.12.008

30. FDA. Guidance for Industry COVID-19: developing Drugs and Biological Products for Treatment or Prevention. 2020. Available from: https://www.fda.gov/regulatory-information/search-fdaguidance-documents/covid-19-developing-drugs-and-biologicalproducts-treatment-or-prevention. Accessed December 16, 2020.

31. Singh AK, Singh A, Singh R, Misra A. Remdesivir in COVID-19: a critical review of pharmacology, pre-clinical and clinical studies. Diabetes Metab Syndr Clin Res Rev. 2020;14(4):641-648. doi:10.1016/j.dsx.2020.05.018

32. Principi N, Esposito S. Chloroquine or hydroxychloroquine for prophylaxis of COVID-19. Lancet Infect Dis. 2020;3099(20):30296. doi:10.1016/S1473-3099(20)30296-6

33. NIBIB. NIH begins study to quantify undetected cases of coronavirus infection. 2020 Available from: https://www.niaid.nih.gov/newsevents/nih-begins-study-quantify-undetected-cases-coronavirusinfection. Accessed March 23, 2020. 
34. Li C, Dockendorf M, Kowalski K. Population PK analyses of ubrogepant (MK-1602), a CGRP receptor antagonist: enriching in-clinic plasma pk sampling with outpatient dried blood spot sampling. J Clin Pharm. 2017;58(3):294-303. doi:10.1002/jcph.1021

35. Lelong C, Wickremsinhe E, Filali-Ansary A, Spooner N. Training and microsample collection. Microsampling Pharm Bioanal. 2013;15-28. doi:10.4155/EBO.13.440

36. FDA. Guide to Informed Consent. 2014. Available From: https:// www.fda.gov/regulatory-information/search-fda-guidance-documents /informed-consent. Accessed December 16, 2020.

37. Moein MM, El Beqqali A, Abdel-Rehim M. Bioanalytical method development and validation: critical concepts and strategies. J Chromatogr B Anal Technol Biomed Life Sci. 2017;1043:3-11. doi:10.1016/j.jchromb.2016.09.028

38. FDA. Bioanalytical Method Validation Guidance for Industry. FDA Guid Ind. 2018:1-22. Available from: http://www.fda.gov/Drugs/ GuidanceComplianceRegulatoryInformation/Guidances/default. htmand/orhttp://www.fda.gov/AnimalVeterinary/ GuidanceComplianceEnforcement/GuidanceforIndustry/default.htm. Accessed December 16, 2020.

39. European Medicines Agency. Guideline on Bioanalytical Method Validation. London: European Medicines Agency; 2011. Available from: https://www.ema.europa.eu/en/documents/scientific-guideline /guideline-bioanalytical-method-validation_en.pdf. Accessed December 16, 2020.

40. Denniff P, Parry S, Dopson W, Spooner N. Quantitative bioanalysis of paracetamol in rats using volumetric absorptive microsampling (VAMS). J Pharm Biomed Anal. 2015;108:61-69. doi:10.1016/j. jpba.2015.01.052

41. Panchal T, Spooner N, Barfield M. Ensuring the collection of high-quality dried blood spot samples across multisite clinical studies. Bioanalysis. 2017;9(2):209-213. doi:10.4155/bio-2016-0189

42. Taylor JM, Hughes AT, Milan AM, Rudge J, Davison AS, Ranganath LR. Evaluation of the Mitra microsampling device for use with key urinary metabolites in patients with Alkaptonuria. Bioanalysis. 2018;10(23):1919-1932. doi:10.4155/bio-2018-0193

43. Li W, Zhang J, Tse FLS. Strategies in quantitative LC-MS/MS analysis of unstable small molecules in biological matrices. Biomed Chromatogr. 2011;25(1):258-277. doi:10.1002/bmc.1572

44. Miao Z, Farnham J, Hanson G, Podoll T, Reid M. Bioanalysis of emixustat (ACU- 4429) in whole blood collected with volumetric absorptive microsampling by LC-MS/MS. Bioanal. 2015;7 (16):2071-2083. doi:10.4155/bio.15.125.

45. Mano Y, Kita K, Kusano K. Hematocrit-independent recovery is a key for bioanalysis using volumetric absorptive microsampling devices, MitraTM. Bioanalysis. 2015;7(15):1821-1829. doi:10.4155/bio.15.111

46. Youhnovski N, Mayrand-Provencher L, Bérubé ER, et al. Volumetric absorptive microsampling combined with impact-assisted extraction for hematocrit effect free assays. Bioanalysis. 2017;9(22):1761-1769. doi:10.4155/bio-2017-0167

47. Spooner N, Denniff P, Michielsen L, et al. A device for dried blood microsampling in quantitative bioanalysis: overcoming the issues associated blood hematocrit. Bioanalysis. 2015;7(6):653-659. doi: $10.4155 /$ bio. 14.310

48. Rudge J, Ranganath LR, Davison AS, Taylor JM, Hughes AT, Milan AM. Evaluation of the Mitra microsampling device for use with key urinary metabolites in patients with Alkaptonuria, Bioanalysis. 1919-1932;10(2018). doi:10.4155/bio-2018-0193

49. De Kesel PMM, Lambert WE, Stove CP. Does volumetric absorptive microsampling eliminate the hematocrit bias for caffeine and paraxanthine in dried blood samples? A comparative study. Anal Chim Acta. 2015;881:65-73. doi:10.1016/j.aca.2015.04.056
50. Parker SL, Jason A, Wallis SC. Quantitative bioanalytical validation of fosfomycin in human whole blood with volumetric absorptive microsampling, Bioanalysis. 2015;7:2585-2595. doi:10.4155/ bio. 15.173

51. Parker SL, Guerra Valero YC, Lipman J, Roberts JA, Wallis SC. Effect of time on recovery of plasma microsamples for the quantitative determination of vancomycin. Bioanalysis. 2016;8:2235-2242. doi:10.4155/bio-2016-0159

52. John H, Willoh S, Hörmann P, Siegert M, Vondran A, Thiermann H, Novel procedures for analysis of dried plasma using microsampling devices to detect sulfur mustard- albumin adducts for verification of poisoning Mitra devices filter paper Noviplex DUO card, (2016). doi:10.1021/acs.analchem.6b02199.

53. Mercolini L, Protti M, Catapano MC, Rudge J, Sberna AE. Journal of Pharmaceutical and Biomedical Analysis LC - MS/MS and volumetric absorptive microsampling for quantitative bioanalysis of cathinone analogues in dried urine, plasma and oral fluid samples. $J$ Pharm Biomed Anal. 2016;123:186-194. doi:10.1016/j. jpba.2016.02.015

54. Ye Z, Gao H. Evaluation of sample extraction methods for minimizing hematocrit effect on whole blood analysis with volumetric absorptive microsampling. Bioanalysis. 2017;9(4):349-357. doi:10.4155/bio-2015-0028

55. Cordell RL, Valkenburg TSE, Pandya HC, et al. Quantitation of salbutamol using micro-volume blood sampling - applications to exacerbations of pediatric asthma. $J$ Asthma. 2017:1-9. doi:10.1080/02770903.2017.1402341

56. Tanna S, Alalaqi A, Bernieh D, Lawson G. Clinical Mass Spectrometry Volumetric absorptive microsampling (VAMS) coupled with high-resolution, accurate-mass (HRAM) mass spectrometry as a simplified alternative to dried blood spot (DBS) analysis for therapeutic drug monitoring of cardiovas, Clin. Mass Spectrom. 2018;10:1-8. doi:10.1016/j.clinms.2018.08.002

57. Kova J, Panic G, Neodo A, et al. Evaluation of a novel microsampling device, Mitra TM, in comparison to dried blood spots, for analysis of praziquantel in Schistosoma haematobium - infected children in rural Côte d ' Ivoire. $J$ Pharm Biomed Anal. 2018;151:339-346. doi:10.1016/j.jpba.2018.01.030

58. Protti M, Carmen M, Gedaliahu B, et al. Journal of Pharmaceutical and Biomedical Analysis Determination of oxycodone and its major metabolites in haematic and urinary matrices: comparison of traditional and miniaturised sampling approaches. J Pharm Biomed Anal. 2018;152:204-214. doi:10.1016/j.jpba.2018.01.043

59. Verheijen RB, Thijssen B, Atrafi F, et al. Validation and clinical application of an LC-MS/MS method for the quantification of everolimus using volumetric absorptive microsampling. J Chromatogr B Anal Technol Biomed Life Sci. 2019;1104:234-239. doi:10.1016/j. jchromb.2018.11.030

60. Marahatta A, Megaraj V, Mcgann PT, Ware RE, Setchell KDR. Stable- isotope dilution HPLC - electrospray ionization tandem mass spectrometry method for quantifying hydroxyurea in dried blood samples. 2016;1601:1593-1601. doi:10.1373/ clinchem.2016.263715

61. Moorthy GS, Vedar C, Zane N, Prodell JL, Zuppa AF. Development and validation of a volumetric absorptive microsampling assay for analysis of voriconazole and voriconazole $\mathrm{N}$-oxide in human whole blood. J Chromatogr B Anal Technol Biomed Life Sci. 2019;1105:67-75. doi:10.1016/j.jchromb.2018.12.007

62. Jones C, Dunseath GJ, Lemon J, Luzio SD. Microsampling collection methods for measurement of C-peptide in whole blood. 2018:1-5. DOI:10.1177/1932296818763464 


\section{Publish your work in this journal}

Drug Design, Development and Therapy is an international, peerreviewed open-access journal that spans the spectrum of drug design and development through to clinical applications. Clinical outcomes, patient safety, and programs for the development and effective, safe, and sustained use of medicines are a feature of the journal, which has also been accepted for indexing on PubMed Central. The manuscript management system is completely online and includes a very quick and fair peer-review system, which is all easy to use. Visit http://www. dovepress.com/testimonials.php to read real quotes from published authors.

Submit your manuscript here: https://www.dovepress.com/drug-design-development-and-therapy-journal 